\title{
The Blue Stellar Objects of the First Byurakan Survey
}

\author{
A.M. Mickaelian \\ Byurakan Astrophysical Observatory (BAO), Byurakan 378433, \\ Armenia. E-mail: aregmick@bao.sci.am
}

\begin{abstract}
The Second part of the FBS is devoted to the discovery and study of blue stellar objects. It was carried out in an area of $4009 \mathrm{deg}^{2}$ and identified 1103 objects: hot subdwarfs and white dwarfs, cataclysmic variables, QSOs and Seyfert galaxies, etc. The discovery of new bright QSOs led to a re-evaluation of their surface density $\left(0.012 \mathrm{deg}^{-2}\right)$. Several interesting cataclysmic variables have been discovered as well.
\end{abstract}

\section{The Second Part of the First Byurakan Survey}

The second part of the First Byurakan Survey (FBS) was conducted in 19871996 for selection and further study of blue stellar objects (BSOs) on the basis of the FBS observational material (Mickaelian 2000). 278 FBS fields (4009 $\mathrm{deg}^{2}$ surface area) have been inspected by eye with $7^{\times}$and $15^{\times}$lenses in the region $+33^{\circ}<\delta<+45^{\circ}$ and $+61^{\circ}<\delta<+90^{\circ}$ (at $|b|>15^{\circ}$ ). The main purpose of this work was discovery of new bright QSOs, Seyferts, other compact galaxies, cataclysmic variables (CV), white dwarfs (WD), hot subdwarfs, HBB stars, and other peculiar stellar objects. 1103 objects have been selected, including 716 new BSOs. In all, 11 lists have been published and the FBS BSOs catalog is available at the CDS (Abrahamian et al. 1999). The completeness of the sample for objects with $\mathrm{B}<16.5^{m}$ and $\mathrm{U}-\mathrm{B}<-0.5$ has been estimated as about $67 \%$. Subsamples of candidate QSOs, WDs, CVs, and other objects have been constructed for further detailed studies.

\section{Bright QSOs and Seyfert Galaxies}

New QSOs have been discovered in the FBS. 15 have been found in the subarea of the FBS with $2250 \mathrm{deg}^{2}$ in common with the Palomar-Green Survey $\left(|b|>30^{\circ}\right)$, substantially changing the existing numbers. In this area, we have constructed the most complete sample for bright QSOs (26 QSOs) (Mickaelian et al. 2001a). The surface density of bright QSOs $\left(\mathrm{B}<16.16^{m}\right)$ is estimated as $0.012 \mathrm{deg}^{-2}$ and the completeness of the Bright Quasar Survey (BQS) (Schmidt \& Green 1983 ) is re-estimated to be $53 \%$. An interesting bright $\left(14^{m}\right)$ very luminous $\left(-24.6^{m}\right)$ NLS1 galaxy FBS $0732+396$ has been found at $\mathrm{z}=0.118$ with strong FeII emission lines.

In all, there are 108 bright $\left(B<17.0^{m}\right)$ AGNs in the FBS subarea with $|b|>30^{\circ}$, including 40 objects discovered in the FBS (Véron-Cetty \& Véron 2001). FBS QSOs and Seyferts have redshifts 0.063-2.00 (QSOs with larger 
redshifts are red enough that it is difficult to distinguish them in the FBS spectra) and absolute B magnitudes in the range $-20.3^{m} \div-29.9^{m}$. NVSS/USNO and ROSAT/USNO cross-correlations, and checking the low-dispersion spectra in FBS with further follow-up spectroscopy identified new QSOs on the FBS plates which had not been selected before, including a new ROSAT NLS1 galaxy RXS $\mathrm{J} 170535+3340$ with $\mathrm{z}=0.118$ having unusual line ratios.

\section{White Dwarfs}

White dwarfs have broad absorption lines and are easily recognized on the FBS plates. Moreover, FBS spectra allow selection of all WDs (not only blue): this task may be completed in the very near future, after the digitization of the FBS. Some subtypes are rather interesting: pulsating white dwarfs (ZZ Ceti stars), magnetic WDs, polars, planetary nebula nuclei (DO stars, PG 1159 type objects), etc.: several objects of these types have been found and dozens are expected to be found. Polarimetric observations revealed $0.7-6.0 \%$ linear polarization for 5 objects, including FBS 1704+347 (a possible polar), and FBS $1815+381$ (a variable magnetic WD). The total number of WDs is estimated to be 270 in the whole sample (24\% of the whole sample). Lists of candidate pulsating and magnetic WDs have been compiled and a detailed study of the most interesting objects has been conducted.

\section{Cataclysmic Variables}

Seven cataclysmic variables have been revealed already from the FBS sample. Two new CVs, FBS 0019+348 and FBS 0306+333, have been observed with the Observatoire de Haute-Provence (OHP) 1.93m telescope in France (in collaboration with M.-P.Véron-Cetty and P.Véron).

A new bright ( $V=12.6)$ cataclysmic variable (Mickaelian et al. 2001b), RXS $\mathrm{J} 16437+3402$, was found by cross-correlation of ROSAT/USNO objects and further inspection of the FBS spectra. Spectroscopic and photometric observations show it to be a novalike cataclysmic variable of the SW Sex subclass. Its spectroscopic period is within the period "gap" for such objects. More observations are needed to describe the physics of this object. The total number of CVs in the FBS sample is estimated to be 35 (3\% of the whole sample). More objects will be found in the total FBS area after the digitization of the survey.

\section{References}

Abrahamian, H.V. et al. 1999, at http://vizier.u-strasbg.fr/

Mickaelian, A.M. 2000, AATr, 18, 557

Mickaelian, A.M. et al. 2001a, Ap, 44, 14

Mickaelian, A.M. et al. 2001b, astro-ph/0108377, A\&A (in press)

Schmidt, M., \& Green, R.F. 1983, ApJ, 269, 352

Véron-Cetty, M.-P. \& Véron, 2001, A\&A, 374, 92 